\title{
Researching Technical Prevention Measures for Development and Design Utilizing Highly-Accuarate CAE Analysis: Automotive Nut-Loosening Mechanism
}

\author{
Ken hashimoto ${ }^{1}$, Kakuro Amasaka ${ }^{2}$ \\ ${ }^{1}$ (Management Technology, Aoyama Gakuin University, Japan) \\ ${ }^{2}$ (Management Technology, Aoyama Gakuin University, Japan)
}

\begin{abstract}
In recent years, the authors have been conducting research on the establishment of technical prevention measures for development and design utilizing highly-accurate CAE analysis. This research investigates the mechanisms that cause nut loosening, which is a matter of concern for automobile manufacturers around the world. Specifically, actual machine tests were conducted using three types of bolt and nut fasteners with different pitches to visualize the dynamic behaviour of changes in axial force, and then experiment analysis of the nut loosening was conducted using an infrared camera. Based on the findings, numerical simulation was conducted incorporating a technical element model for highly-accurate CAE analysis. Thus, a highly-reliable CAE analysis approach model was constructed, which enables intelligent development and design to be achieved by eliminating discrepancies between the results of the actual machine tests and the results of the CAE analysis. This approach model contributes to creating technical prevention measures that lead to product design using predictive technical evaluation.
\end{abstract}

Keywords: CAE, Nut loosening Mechanism, Simulation, technical element model

\section{Introduction}

In recent years, the authors have been conducting research on the establishment of technical prevention measures for development and design utilizing highly-accurate CAE analysis. This research investigates the mechanisms that cause nut loosening, which is a matter of concern for automobile manufacturers around the world. Specifically, actual machine tests were conducted using three types of bolt and nut fasteners with different pitches to visualize the dynamic behavior of changes in axial force, and then experiment analysis of the nut loosening was conducted using an infrared camera. Based on the findings, numerical simulation was conducted incorporating a technical element model for highly-accurate CAE analysis. Thus, a highly-reliable CAE analysis approach model was constructed, which enables intelligent development and design to be achieved by eliminating discrepancies between the results of the actual machine tests and the results of the CAE analysis. This approach model contributes to creating technical prevention measures that lead to product design using predictive technical evaluation.

\section{Developing A Highly Reliable Cae Analysis Approach Model}

\subsection{Highly Reliable CAE Analysis}

Highly reliable CAE analysis, meaning CAE analysis for which there is no gap between its results and the results of actual machine tests, allows the realization of intelligent development and design. Specifically, the development of an approach that implements highly reliable CAE analysis contributes to the creation of drawings in manufacturing design by allowing the realization of specific creative support for developers and designers.

\subsection{Developing a Highly Reliable CAE Analysis Approach Model}

In this study, we focused on the loosening of nut fasteners to develop a highly reliable CAE analysis approach model using the action elements described below in Steps 1 through 5, summarized in Fig. 1.

(1) Assessment of the phenomenon (Step 1): As a preliminary investigation, we defined the problem by asking what issues are occurring based on the details of past accidents and what mechanisms need to be explained. In this step, the authors pursued cooperative creative activities that bring together the knowledge of both in-house and outside experts.The authors used the latest techniques of statistical science to investigate and analyze convergent causal relationships and conduct a detailed analysis of the phenomenon in question in order to deduce the failure mechanism.

(2) Tests to visualize the phenomenon (Step 2): In order to visualize the failure mechanism, it was necessary to make clear the dynamic behavior that accompanies occurrence of the problem using the visualization technologies that are best suited to the task and a program of tests using the actual machine in question. 
Specifically, use the techniques of statistical science, including N7, SQC, RE (a reliability technique), MA (multivariate analysis), and DE (design of experiments) to accurately analyze the failure and its cause. Then search for latent factors that have remained unknown or overlooked and assess the failure mechanism by means of logical thought processes.

(3) Agreement between actual machine tests and CAE (Step 3): Drawing on the information obtained in Steps 1 and 2, we incorporated the principal factors in the occurrence of the failure (technical elements) into a numerical simulation and pursued a highly accurate CAE analysis in which there were no gaps between actual machine tests and the CAE analysis. Specifically, we devised a three-dimensional model using CAD (3D). At this stage, all business processes in the technical element model (phenomenon assessment, modeling, algorithms, theory, and computer technology) that should be incorporated into highly accurate CAE analysis in a scientific and comprehensive manner were optimized. To perform a highly accurate numerical simulation, the above should be combined with an experimental model capable of rationally identifying the effects of the latent factors that were discovered using precise theory and tests. At this point, it is important to select the model in order to rationalize calculation techniques, analytical models, and algorithms, and the task is to identify and model the failure mechanism.

(4) Highly Accurate CAE analysis (Step 4): During highly accurate CAE analysis, we developed a threedimensional model based on the information gained in Step 3 and articulated implicit knowledge about the failure mechanism. The authors integrated the information obtained from these processes, conducted a highly reliable numerical simulation that enables the prediction and control of the absolute values needed for the CAE analysis, and implemented predictive technical evaluation-type CAE analysis.

(5) Prediction and evaluation (Step 5): In predictive technical evaluation-type CAE analysis that enables highly accurate prediction and evaluation, since it is possible to identify principal factors, we accurately assess the development and design factors that should be subject to optimal control, and apply them to drawings and manufacturing techniques as articulated knowledge. The authors then conducted product design quickly and under optimal conditions so as to verify the validity of this approach model.

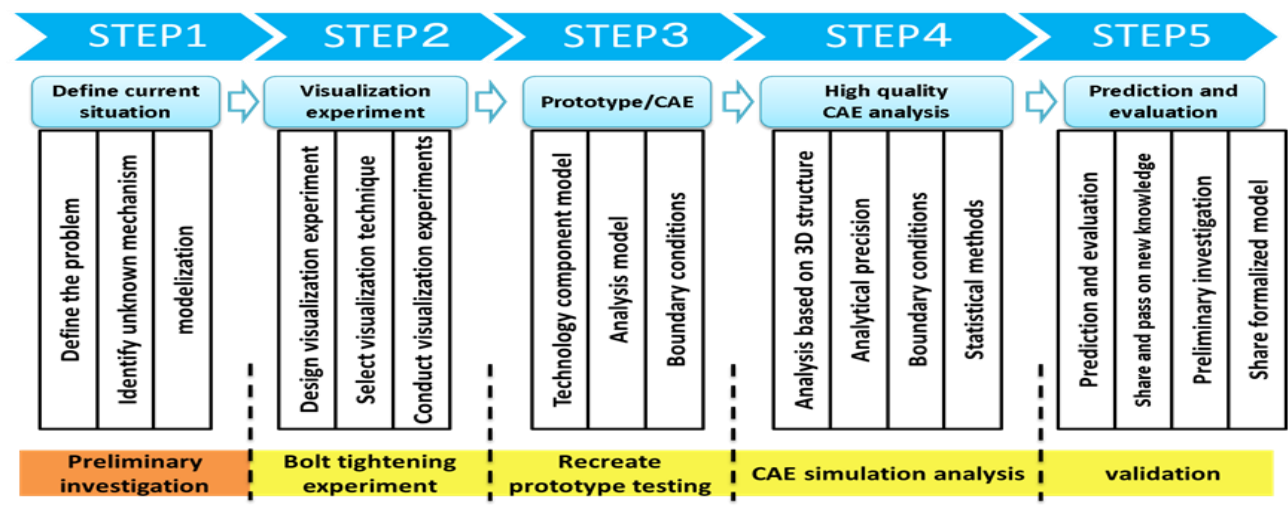

Fig. 1. Highly Reliable CAE Analysis Approach Model

\section{Example Applicationa-Analysis Of Mechanisms Causing Bolt Loosening In Automobiles}

The authors applied the highly reliable CAE approach model to analyze the mechanisms causing bolt loosening in automobiles. Specifically, since past research had already established that loosening occurs from the nut portion of bolt and nut fasteners, this study focused exclusively on nuts in its analysis. In terms of our experimental method, we used an infrared camera to conduct a stress analysis of nut looseness and used the technical element model for highly accurate CAE analysis to conduct a numerical simulation based on the information we obtained.

\subsection{Bolt Fastening Test}

We conducted a bolt fastening test using two bolts: one with a pitch of $0.50 \mathrm{~mm}$ (see Fig. 2. (a)) and one with a pitch of $1.75 \mathrm{~mm}$ (see Fig. 2. (b)), the smallest and largest pitches, respectively, that can be manufactured outside the JIS standard for flanged hexagonal bolts and nuts. First, in order to estimate the sliding load to be used in a vibration test, we applied a load to bolt fasteners that had been fastened with fastening loads of $20 \mathrm{kN}$ and $35 \mathrm{kN}$ as a static test and measured the tester load at which sliding of the seating surface increased abruptly. Specifically, we induced vibration displacement by applying an external force in the direction of the plane perpendicular to the bolts' axis, measured the nuts' fastening load, and verified the change in fastening load relative to the displacement. We then applied vibration with the tester at a vibration load equal to $\pm 90 \%$ of the static extraction load. By repeatedly inducing sliding of the nut seating surface, we were able to assess the phenomenon that causes nuts to loosen. We then measured bolt axial force/tester displacement and nut fastening load relative to vibration time as changes in temperature and nut fastening load using an infrared camera. 

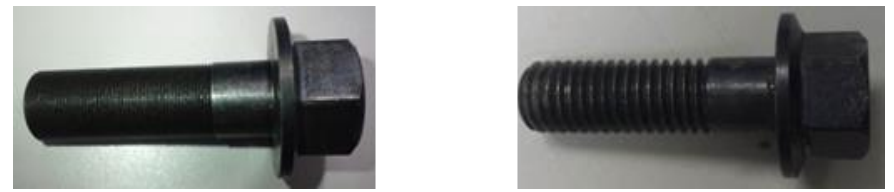

Fig. 2. (a) pitch of $0.50 \mathrm{~mm}$; (b) pitch of $1.75 \mathrm{~mm}$

3.2 Numerical Simulation

\subsubsection{Creating a Technical Element Model}

To facilitate highly accurate CAE analysis, the authors developed a nut fastener technical element model based on five principal factors in order to allow accurate reproduction of actual machine tests (see Fig.3.). This model addresses the failure mechanism through steps (1) to (5) below.

(1) Assessment of the phenomenon: This step consists of clarifying the problematic causal relationship and assessing the phenomenon through actual machine tests. We assessed the phenomenon by means of actual machine tests employing the Second Law of Thermodynamics, interpreting temperature changes detected with an infrared camera as stress changes.

(2) Modeling: To convert the entire nut being targeted in our analysis into a model that can work with CAE analysis, we broke it down into elements and modeled the elements' material constitutive principle model as an equation.

(3) Algorithms: We synchronized important algorithms (calculation procedures) being used as analytical means with locations where the effects of external force were observed and eliminated gaps with actual machine tests through repetition so that the accuracy of our finite element method analysis and nonlinear analysis would increase.

(4) Theory: Since the validity, application scope, and performance of the algorithms themselves can be derived from theory, we calculated coefficients of friction using experimental values and a theoretical equation. Furthermore, we calculated these coefficients of friction, which vary with temperature, accurately by holding the temperature constant utilizing an infrared camera.

(5) Computer technology: Computer technology (computational techniques) plays the role of actually calculating the algorithms, and we used the penalty method, which is characterized by numerical stability and a tendency to converge on solutions, to formulate the area of contact identified in (1) with the highest impact.

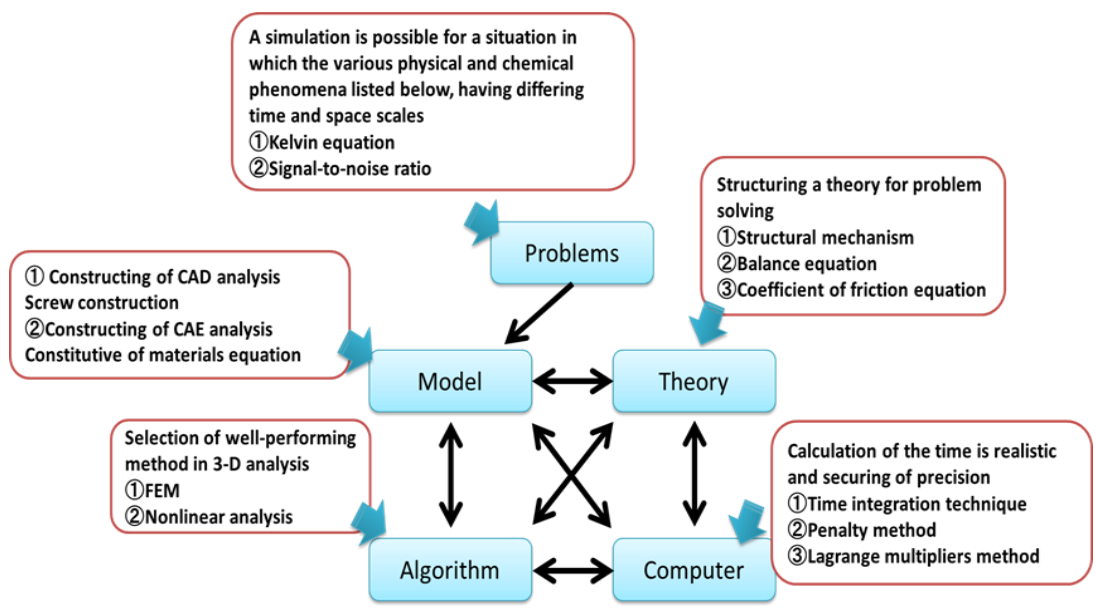

Fig. 3. Technical Element Model

\subsubsection{Three-dimensional CAE Analysis of the Bolt Fastening Area}

In our CAE analysis of the bolt and nut fasteners, we assessed the stress to which the entire fastener was subjected by performing a finite element method analysis of a three-dimensional model that took into account the thread's helical structure. This analysis was performed by means of the following steps. First, we placed the objects being fastened (two sheets of base material) between the bolts and nuts. Second, we applied axial force to the bolts and force (axial force) to the vertical base material. Third, we calculated the contact surface pressure (between the bolt and nut seating surface and the base material, between the bolt thread and the nut, and at the nut's air contact surface) as well as the behavior in which the axial force drops at that time. Fig. 4. (a) and Fig. 4. (b) illustrate the results of an analysis in which axial force was applied to the bolt fasteners and an axial force extraction load was applied in the plane perpendicular to the bolt axis. The figures reveal that the maximum stress occurs at the beginning of the thread's helix. Next, looking at different pitches, stress that is 
concentrated at the beginning of the thread on the $1.75 \mathrm{~mm}$ pitch bolt is distributed. Non-uniformity varies throughout the nut due to the pitch difference, and the results show differences in areas of varying levels of stress. As a novel finding, we were able to confirm that the bolt and nut pitch affects the non-uniformity of stress throughout the nut.
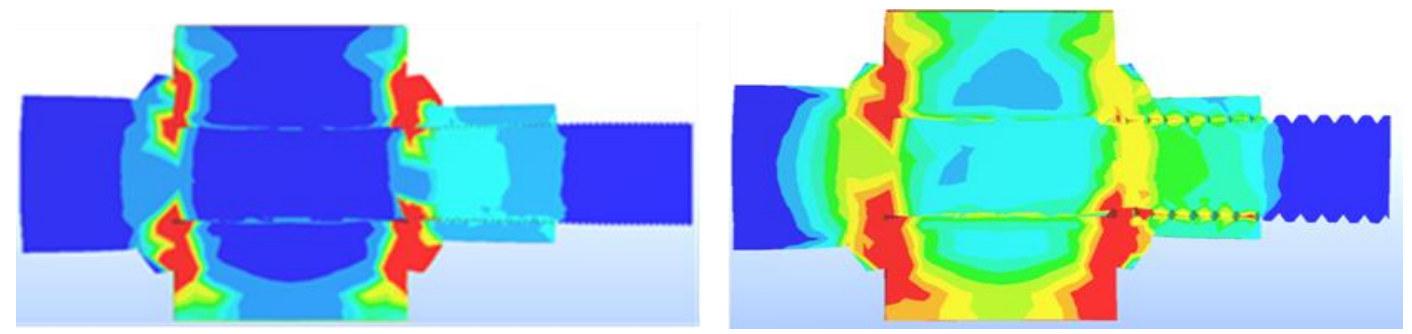

Fig. 4. (a) result of CAE analysis (pitch of $0.50 \mathrm{~mm}$ ) ; (b) result of CAE analysis (pitch of $1.75 \mathrm{~mm}$ )

\subsection{Verification of the Three-dimensional CAE Analysis}

Similarly, we verified the accuracy of the CAE analysis results by using an infrared camera to measure stress at the contact surface between the nut and the base material and comparing the findings with the results of our analysis. The infrared camera treats temperature changes caused by vibrations as stress, which can be displayed as contour colors and values. Fig. 5. (a) illustrates the stress measurement results for the $1.75 \mathrm{~mm}$ pitch nut, while Fig. 5. (b) illustrates the results of the CAE analysis simulation for the same nut. Both comprise stress measurement results when an axial force extraction load was applied to axial force. The infrared camera image shows maximum stress occurring in the area near the beginning of the thread's helix, and the numerical analysis simulation results shown in Fig. 5. (b) Reproduce this phenomenon.
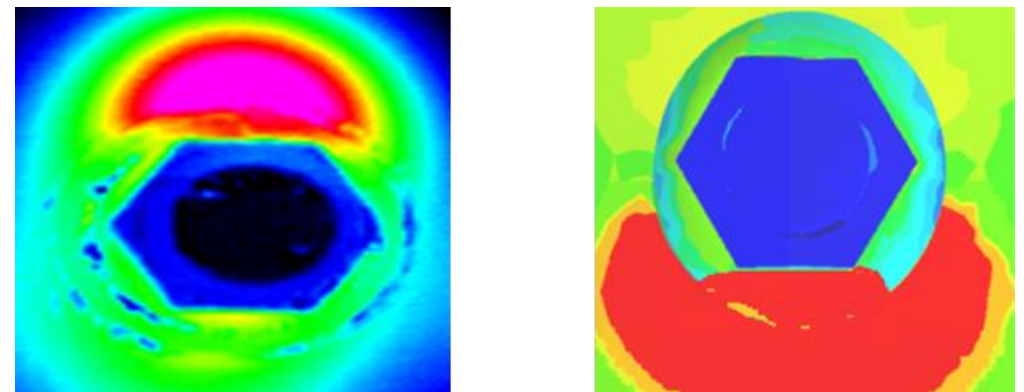

Fig. 5. (a) result of infrared camera; (b) result of CAE analysis

\section{Conclusion}

The authors developed a highly reliable CAE analysis approach model to contribute specific creative support to developers and designers, applied the model to an analysis of stress in bolt fasteners, which is an area of concern, and obtained the expected results.

\section{References}

[1] Amasaka K, Yamaji M. CAE Analysis Technology for Development Design Utilizing Statistical Sciences. The Open Industrial and Manufacturing Engineering Journal 2008;1:1-8

[2] Amasaka K. Proposal and Effectiveness of a High Quality Assurance CAE Analysis Model: Innovation of Design and Development in Automotive Industry. Current Development in Theory and Applications of Computer Science: Engineering and Technology 2010;2:1/2, 23-48

[3] Yamada H, Amasaka K. Highly-Reliable CAE Analysis Approach-Application in Automotive Bolt Analysis. China-USA Business Review 2011;10:3, 199-205

[4] Onodera T, Amasaka K. A Study on a High Quality CAE Analysis Approach Model -Automotive Bolts Tightening Analysis using Contact Stress Simulation-. Proceedings of International Symposium on System Integration, Kyoto;2011,p. 1379-1382

[5] Onodera T, Amasaka K. Automotive Bolts Tightening Analysis using Contact Stress Simulation: Developing an Optimal CAE Design Approach Model. Journal of Business \& Economics Research 2012;10:7,435-442 